\title{
THE INDEX OF SYMMETRY OF COMPACT NATURALLY REDUCTIVE SPACES
}

\author{
CARLOS OLMOS, SILVIO REGGIANI, AND HIROSHI TAMARU
}

\begin{abstract}
We introduce a geometric invariant that we call the index of symmetry, which measures how far is a Riemannian manifold from being a symmetric space. We compute, in a geometric way, the index of symmetry of compact naturally reductive spaces. In this case, the so-called leaf of symmetry turns out to be of the group type. We also study several examples where the leaf of symmetry is not of the group type. Interesting examples arise from the unit tangent bundle of the sphere of curvature 2, and two metrics in an Aloff-Wallach 7-manifold and the Wallach 24-manifold.
\end{abstract}

\section{INTRODUCTION}

The study of Riemannian homogeneous spaces is a very important research area inside Riemannian geometry. The symmetric spaces, defined and classified by É. Cartan [Car26], are a distinguished family among all homogeneous spaces. The symmetric spaces can be defined in several ways. For example, these spaces are locally characterized by the property of having parallel curvature tensor, or globally, by the fact that the geodesic symmetry at any point extends to a global isometry. Another way of defining a symmetric space $M$ is the following. Given $p \in M$ and $v \in T_{p} M$ there exists a Killing field $X$ on $M$ such that $X(p)=v$ and $(\nabla X)_{p}=0$. That is, there is a Killing field, in any direction, which is parallel at $p$.

The symmetric spaces generalize, in a natural way, to larger families of homogeneous spaces. This is the case of naturally reductive spaces. Recall that in a naturally reductive space $M=G / H$ there always exists a canonical connection $\nabla^{c}$, which is $G$-invariant and has the same geodesics as the Levi-Civita connection (and hence, $\nabla^{c}$ has totally skew-symmetric torsion). In particular, the Riemannian curvature tensor is parallel with respect to the canonical connection, $\nabla^{c} R=0$. For a symmetric space, the Levi-Civita connection is a canonical connection.

In recent years, some relevant results on naturally reductive spaces have been obtained in the framework of connections with skew-symmetric torsion. In particular, it is proved that the canonical connection of a naturally reductive space is essentially unique OR12a, OR12b (except for certain well-studied cases, which are all symmetric).

Notice that the family of naturally reductive spaces contains the compact isotropy irreducible spaces and, more generally, the normal homogeneous spaces.

2010 Mathematics Subject Classification. Primary 53C30; Secondary 53C35.

Key words and phrases. Index of symmetry, distribution of symmetry, naturally reductive space, symmetric space.

The work of C. Olmos and S. Reggiani was supported by Universidad Nacional de Córdoba and CONICET, and partially supported by ANCyT, Secyt-UNC and CIEM. H. Tamaru was supported in part by KAKENHI (24654012). 
In this article we deal with a geometric invariant $0 \leq i_{\mathfrak{s}}(M) \leq \operatorname{dim} M$, that we call the index of symmetry of the Riemannian manifold $M$. Roughly speaking, the index of symmetry of $M$ measures how far is $M$ from being a symmetric space, in the sense that $M$ is symmetric if and only if $i_{\mathfrak{s}}(M)=\operatorname{dim} M$. The index of symmetry of $M$ can be defined as follows: it is the greatest non-negative integer $k$ such that at every $p \in M$ there exist at least $k$ linear independent vectors $v_{1}, \ldots, v_{k} \in T_{p} M$ and $k$ Killing fields $X_{1}, \ldots, X_{k}$ on $M$ such that $X_{i}(p)=v_{i}$ and $\left(\nabla X_{i}\right)_{p}=0$.

If $i_{\mathfrak{s}}(M)=k$, given $p \in M$, one can consider the subspace

$$
\mathfrak{s}_{p}=\left\{X(p): X \text { is a Killing field on } M \text { with }(\nabla X)_{p}=0\right\} \subset T_{p} M,
$$

which is a subspace of dimension at least $k$. So, $p \mapsto \mathfrak{s}_{p}$ defines an, a priori nonsmooth nor even of constant dimension, distribution $\mathfrak{s}$ on $M$. The distribution $\mathfrak{s}$ is called the distribution of symmetry of $M$.

If $M$ is a Riemannian homogeneous space, we have that the distribution of symmetry has constant dimension and it is smooth (since it is invariant under the full isometry group of $M$ ). Hence $i_{\mathfrak{s}}(M)=\operatorname{dim} \mathfrak{s}_{p}$ for any $p \in M$. We prove that $\mathfrak{s}$ is an integrable distribution with totally geodesic leaves (or equivalently, $\mathfrak{s}$ is autoparallel). Moreover, the leaves of $\mathfrak{s}$ turn out to be intrinsically globally symmetric submanifolds of $M$.

The main goal of this article is to compute the index of symmetry of compact normal homogeneous spaces. More generally, we explicitly determine the distribution of symmetry for these spaces. Namely,

Theorem A. Let $M=G / H$ be a simply connected compact normal homogeneous space, where $G$ is connected. Let us assume that $M$ is an irreducible Riemannian manifold which is not a symmetric space. Then the distribution of symmetry of $M$ coincides with the $G$-invariant distribution defined by the fixed vectors of $H$.

The above theorem is still true for compact naturally reductive spaces under the hypothesis that the transitive presentation group $G$ is the transvection group of the canonical connection. Namely,

Theorem B. Let $M=G / H$ be a simply connected compact naturally reductive space, where $G$ is the group of transvections of the canonical connection. Let us assume that $M$ is an irreducible Riemannian manifold which is not a symmetric space. Then the distribution of symmetry of $M$ coincides with the $G$-invariant distribution defined by the fixed vectors of $H$.

In order to prove Theorem $\mathrm{A}$, we deal with the de Rham decomposition of the leaf of symmetry $L(p)$ of $\mathfrak{s}$ at an arbitrary point $p$. We first prove that the (connected component by $p$ ) of the fixed points of $H$ in $M, \Sigma$, must be contained in $L(p)$. Moreover, we show that the flat factor of $L(p)$ by $p$ must be inside $\Sigma$. Then, we finally prove that the semisimple factors of $L(p)$ must be tangent to $\Sigma$. The proof depends on general arguments and uses strongly Proposition 3.6 and the precise knowledge of the full isometry group (see Reg10, OR12a, OR12b]).

For proving Theorem B we make use of the so-called Kostant bilinear form, which allows us to think of a naturally reductive space as a normal homogeneous space with respect to a bi-invariant pseudo-Riemannian metric defined on the group of presentation. In this case, the presentation group must be the group of transvection of the canonical connection. Similar arguments, as for the normal homogeneous case, work. (Though it is not trivial to adapt some of the arguments.) 
Recall, as it follows from Theorems $\mathrm{A}$ and $\mathrm{B}$, that for naturally reductive spaces the leaf of symmetry is of the group type. We finish the article by giving several examples of compact homogeneous spaces with non-trivial index of symmetry and such that the leaf of symmetry is not of the group type. To do this, we work with triples $G \supset G^{\prime} \supset K^{\prime}$, where $G / G^{\prime}$ and $G^{\prime} / K^{\prime}$ are symmetric spaces. The examples arise by perturbing the normal homogeneous metric on $G / K^{\prime}$. Interesting examples, obtained in this way, are the unit tangent bundle of the sphere of curvature 2, and two metrics that occur in the Aloff-Wallach manifold $W_{1,-1}^{7}=\mathrm{SU}(3) / \mathrm{SO}(2)$ and the Wallach manifold $W^{24}=F_{4} / \operatorname{Spin}(8)$.

This article can be regarded as an effort to understand, in a geometric way, naturally reductive spaces. This is in the same spirit of the articles OR12a, Reg10, OR12b, Reg13.

\section{PRELIMINARIES AND BASIC FACTS}

2.1. Infinitesimal transvections. Let $M$ be a Riemannian manifold. Throughout this article we will call an infinitesimal transvection, or just a (geometric) transvection, at $p \in M$, to a Killing vector field $X$ such that $(\nabla X)_{q}=0$, where $\nabla$ is the Levi-Civita connection. Recall that $M^{n}$ a symmetric space if and only if for all $p \in M$ there exist transvections $X_{1}, \ldots, X_{n}$, at $p$, such that $X_{1}(p), \ldots, X_{n}(p)$ is a basis of $T_{p} M$.

Do not confuse the infinitesimal transvection with the transvection of the canonical connection of a naturally reductive space. In fact, let $M=G / H$ be a naturally reductive space with associated reductive decomposition $\mathfrak{g}=\mathfrak{h} \oplus \mathfrak{m}$. That is, $M$ carries a $G$-invariant metric, $\mathfrak{g}$ is the Lie algebra of $G, \mathfrak{h}$ is the Lie algebra of $H$ and $\mathfrak{m}$ is an $\operatorname{Ad}(H)$-invariant subspace such that the geodesics through $p=e H$ are given by $\operatorname{Exp}(t X) \cdot p, X \in \mathfrak{m}$. Let $\nabla^{c}$ be the canonical connection of $M$ (i.e., the $G$ invariant connection associated with the decomposition $\mathfrak{g}=\mathfrak{h} \oplus \mathfrak{m})$. The Lie algebra of transvections of $\nabla^{c}$ is $\mathfrak{t r}\left(M, \nabla^{c}\right)=\mathfrak{m}+[\mathfrak{m}, \mathfrak{m}]$, and the connected associated Lie subgroup is $\operatorname{Tr}\left(M, \nabla^{c}\right)$. Recall that $\operatorname{Tr}\left(M, \nabla^{c}\right)$ is a transitive normal subgroup of $G$. When the metric on $M$ is also normal homogeneous, then $\operatorname{Tr}\left(M, \nabla^{c}\right)$ coincides with connected component of $G$ (see Reg10]).

Of course, a transvection of the canonical connections needs not to be a geometric transvection.

2.2. Ad-invariant bilinear forms. In this subsection we want to point out the following elementary remark, which will be very useful in the sequel.

Remark 2.1. Let $\mathfrak{g}$ be a Lie algebra and $Q$ an Ad-invariant symmetric bilinear form on $\mathfrak{g}$. Assume that $\mathfrak{g}$ is the sum of the ideals $\mathfrak{g}=\mathfrak{g}_{1} \oplus \mathfrak{g}_{2}$ where $\mathfrak{g}_{1}$ is semisimple. Then, such a decomposition must be orthogonal with respect to $Q$, that is $Q\left(\mathfrak{g}_{1}, \mathfrak{g}_{2}\right)=0$. Moreover, if $\mathfrak{g}_{1}$ is simple, then the restriction $\left.Q\right|_{\mathfrak{g}_{1}}$ of $Q$ to the ideal $\mathfrak{g}_{1}$ must be a scalar multiple of the Killing form of $\mathfrak{g}_{1}$.

In fact, let $X^{\prime}, X^{\prime \prime} \in \mathfrak{g}_{1}$ and $Y \in \mathfrak{g}_{2}$. If $X=\left[X^{\prime}, X^{\prime \prime}\right]$, an standard calculation gives

$$
Q(X, Y)=Q\left(\left[X^{\prime}, X^{\prime \prime}\right], Y\right)=-Q\left(X^{\prime \prime},\left[X^{\prime}, Y\right]\right)=-Q\left(X^{\prime \prime}, 0\right)=0 .
$$

Since $\mathfrak{g}_{1}$ is semisimple, it is linearly spanned by elements of the form $X=\left[X^{\prime}, X^{\prime \prime}\right]$, and therefore $Q\left(\mathfrak{g}_{1}, \mathfrak{g}_{2}\right)=0$.

If we also assume that $\mathfrak{g}_{1}$ is simple, it is well known that Schur's Lemma implies that $\left.Q\right|_{\mathfrak{g}_{1}}$ is a multiple of the Killing form of $\mathfrak{g}_{1}$. 
2.3. The isometry group of naturally reductive spaces. Let $M=G / H$ be a compact and locally irreducible naturally reductive space. Let $\nabla^{c}$ be the canonical connection on $M$. In OR12a it is proved that the connected component of the group $\operatorname{Aff}_{0}\left(M, \nabla^{c}\right)$ of $\nabla^{c}$-affine transformations coincides with the connected component of the full isometry group of $M$, except for spheres or real projective spaces. On the other hand, in Reg10 it is studied the structure of $\operatorname{Aff}_{0}\left(M, \nabla^{c}\right)$ when $M$ is also normal homogeneous. This gives the following characterization of the (connected component of the) isometry group of a normal homogeneous space.

Theorem 2.2 (see Reg10). Let $M=G / H$ be a compact normal homogeneous space. Assume that $M$ is locally irreducible and that it is neither (globally) isometric to a sphere, nor to a real projective space. Write $G=G_{\mathrm{ab}} \times G_{\mathrm{ss}}$ as an almost direct product, where $G_{\mathrm{ab}}$ is abelian and $G_{\mathrm{ss}}$ is a semisimple Lie group of the compact type. Then

$$
I_{0}(M)=G_{\mathrm{ss}} \times F \quad \text { (almost direct product), }
$$

where $F$ is the connected component by $p=e H$ of the fixed points of $H$ (regarded as a Lie group).

Recall that the Lie algebra of $F$ may be identified with the Lie algebra of $G$ invariant fields on $M$.

Actually, Theorem 2.2 remains true if we assume that $M$ is naturally reductive. In fact, the key factor to prove Theorem 2.2 in Reg10 is that $G$-invariant fields are Killing fields. This is also true if $M$ is naturally reductive. In fact, the difference tensor $D=\nabla-\nabla^{c}$, where $\nabla$ is the Levi-Civita connection of $M$, is totally skew-symmetric. If $X$ is a $G$-invariant field, then $\nabla^{c} X=0$ (since the canonical connection is $G$-invariant). Then

$$
\nabla X=\nabla X-\nabla^{c} X=D X
$$

is skew-symmetric, and hence $X$ is a Killing field. Therefore, the proof of Theorem 2.2 given in Reg10 also works in the naturally reductive case (see [OR12b]).

\section{The InDEX OF SYMMeTRY}

Let $M^{n}$ be a Riemannian manifold and denote by $\mathfrak{K}(M)$ the Lie algebra of global Killing fields on $M$. For $q \in M$, let us define the Cartan subspace $\mathfrak{p}^{q}$ at $q$ by

$$
\mathfrak{p}^{q}:=\left\{X \in \mathfrak{K}(M):(\nabla X)_{q}=0\right\} .
$$

The symmetric isotropy algebra at $q$ is defined by

$$
\mathfrak{k}^{q}:=\left\{[X, Y]: X, Y \in \mathfrak{p}^{q}\right\} .
$$

Observe that $\mathfrak{k}^{q}$ is contained in the (full) isotropy subalgebra $\mathfrak{K}_{q}(M)$. In fact, if $X, Y \in \mathfrak{p}^{q}$, then $[X, Y]_{q}=\left(\nabla_{X} Y\right)_{q}-\left(\nabla_{Y} X\right)_{q}=0$. Moreover, since $\mathfrak{p}^{q}$ is left invariant by the isotropy at $q$,

is an involutive Lie algebra.

$$
\mathfrak{g}^{q}:=\mathfrak{k}^{q} \oplus \mathfrak{p}^{q}
$$

Remark 3.1. If $X \in \mathfrak{p}^{q}$, then $\gamma(t)=\operatorname{Exp}(t X) \cdot q$ is a geodesic. Moreover, the parallel transport along $\gamma(t)$ is given by $\left.d m_{\operatorname{Exp}(t X)}\right|_{q}$, where $m_{g}(x)=g \cdot x$. In fact, for any Killing field $X$,

$$
\left.\tau_{-t} \circ d m_{\operatorname{Exp}(t X)}\right|_{q}=e^{t(\nabla X)_{q}} \in \mathfrak{s o}\left(T_{q} M\right),
$$

where $\tau_{t}$ is the parallel transport along the curve $\operatorname{Exp}(t X) \cdot q$ (see [BCO03, p. 163]). 
Let $G^{q}$ be the Lie subgroup, of the full isometry group $I(M)$, associated to the Lie algebra $\mathfrak{g}^{q}$. By the previous remark, the orbit $G^{q} \cdot q$ is a totally geodesic submanifold of $M$.

Observe that $G^{g \cdot q}=g \cdot G^{q} \cdot g^{-1}$ and that $G^{x}=G^{q}$, for all $x \in G^{q} \cdot q$.

The symmetric subspace at $q, \mathfrak{s}_{q} \subset T_{q} M$, is defined by

$$
\mathfrak{s}_{q}:=\left\{X \cdot q: X \in \mathfrak{p}^{q}\right\}=\mathfrak{p}^{q} \cdot q .
$$

Definition 3.2. The index of symmetry $i_{\mathfrak{s}}(M)$ of a Riemannian manifold $M$ is the infimum, over $q \in M$, of the dimensions of $\mathfrak{s}_{q}$.

Observe that

$$
L(q):=G^{q} \cdot q=\exp \left(\mathfrak{s}_{q}\right) .
$$

Moreover, for any $x \in G^{q} \cdot q, T_{x}\left(G^{q} \cdot q\right)=\mathfrak{s}_{x}$. So, the totally geodesic submanifold $L(q)$ is a leaf of the a priori non necessarily smooth (and eventually singular) distribution $p \mapsto \mathfrak{s}_{p}, p \in M$. Note that $L(q)$ is a locally symmetric space, whose group of transvections is $G^{q}$, quotioned by the ideal of elements that acts trivially on $L(q)$ (we will see later that $G^{q}$ acts almost effectively on $L(q)$ if $M$ is compact). Moreover, $L(q)$ is a globally symmetric space, as follows from Theorem A of EO94] (see also Lemma 5 of this reference).

Lemma 3.3. If $M$ is compact then $G^{q}$ acts almost effectively on $L(q)=G^{q} \cdot q$, for all $q \in M$.

Proof. Since $M$ is compact, the isometry group $I(M)$ is compact. Let $(\cdot, \cdot)$ be an $\operatorname{Ad}(I(M))$-invariant inner product on the Lie algebra of $I(M)$.

Let $\mathfrak{h} \subset \mathfrak{k}^{q}$ the ideal which corresponds to the normal subgroup of $H$ of $G^{q}$ that acts trivially on $L(q)$. Let $Z \in \mathfrak{h}$. Then $\left[Z, \mathfrak{p}^{q}\right] \subset \mathfrak{p}^{q}$, since the isotropy at $q$ leaves invariant $\mathfrak{p}^{q}$. On the other hand, since $H$ acts trivially on $T_{q}(L(q))=\mathfrak{s}_{q}$, one has that $[Z, X]_{q}=0$, for all $X \in \mathfrak{p}^{q}$. Then $\left[Z, \mathfrak{p}^{q}\right]=\{0\}$. Therefore, if $X, Y \in \mathfrak{p}^{q}$, $(Z,[X, Y])=-([Z, X], Y)=0$. Thus, $Z$ is perpendicular to $\left[\mathfrak{p}^{q}, \mathfrak{p}^{q}\right]=\mathfrak{k}^{q}$. Hence $\mathfrak{h}=\{0\}$.

We identify $T_{q}\left(G^{q} \cdot q\right)=\mathfrak{s}_{q} \simeq \mathfrak{p}^{q}$ and decompose $\mathfrak{p}^{q}=\mathfrak{p}_{0}^{q} \oplus \mathfrak{p}_{1}^{q} \oplus \cdots \oplus \mathfrak{p}_{s}^{q}$, where $\mathfrak{p}_{0}^{q}$ corresponds to the Euclidean factor and $\mathfrak{p}_{i}^{q}, i \geq 1$, corresponds to the irreducible $i$-th factor in the de Rham decomposition of $L(q)=G^{q} \cdot q$. Let $\mathfrak{k}_{j}^{q}=\left[\mathfrak{p}_{j}^{q}, \mathfrak{p}_{j}^{q}\right]$ for $j=0, \ldots, s$ and let $\mathfrak{g}_{j}^{q}=\mathfrak{k}_{j}^{q} \oplus \mathfrak{p}_{j}^{q}$.

Corollary 3.4. If $M$ is compact then $\mathfrak{k}_{0}^{q}=\{0\}$, $\left[\mathfrak{g}_{i}^{q}, \mathfrak{g}_{j}^{q}\right]=\{0\}$, if $i \neq j$ and so $\mathfrak{g}^{q}$ is the direct sum of the ideals $\mathfrak{g}_{1}^{q}, \ldots, \mathfrak{g}_{s}^{q}$. Then

$$
G^{q}=G_{0}^{q} \times \cdots \times G_{s}^{q} \quad \text { (almost direct product), }
$$

where $\operatorname{Lie}\left(G_{i}^{q}\right)=\mathfrak{g}_{i}^{q}$.

In the notation of the above corollary, we denote

$$
L_{i}(q):=G_{i}^{q} \cdot q, \quad i=1, \ldots, s,
$$

and we call $L_{i}(q)$ the $i$-th de Rham factor by $q$ of $L(q)$.

More generally, if $J \subset\{0, \ldots, s\}$ we denote by

$$
G_{J}^{q}=\prod_{j \in J} G_{j}^{q} .
$$

The orbit $L_{J}(q):=G_{J}^{q} \cdot q$ is called a local factor by $q$ of $L(q)$. 
Let

$$
\tilde{G}^{q}=\{g \in I(M): g \cdot L(q)=L(q)\}_{0} .
$$

Let $g \in \tilde{G}^{q}$, then $G^{q}=G^{g \cdot q}=g G^{q} g^{-1}$. So, $G^{q}$ is a normal subgroup of $\tilde{G}^{q}$.

Let

$$
\bar{H}^{q}=\{g \in I(M): g \text { acts trivially on } L(q)\}_{0} .
$$

Observe that $\bar{H}^{q}$ is also a normal subgroup of $\tilde{G}^{q}$.

Lemma 3.5. $\tilde{G}^{q}=G^{q} \times \bar{H}^{q}$ (almost direct product).

Proof. By Lemma 3.3, $G^{q} \cap \bar{H}^{q}$ is discrete. Let $X$ be a Killing field induced by $\tilde{G}^{q}$. Then $\left.X\right|_{L(q)}$ is an intrinsic Killing field of $L(q)$ which is bounded. Then it must lie in the Lie algebra of the (intrinsic) transvection group of $L(q)$. Then there exists a Killing field $Y \in \mathfrak{g}^{q}$ such that $\left.Y\right|_{L(q)}=\left.X\right|_{L(q)}$. Then $Z=Y-X$ is null when restricted to $L(q)$. Then $Z$ lies in the Lie algebra of $\bar{H}^{q}$. This implies the desired decomposition.

We denote, if $J \subset\{0, \ldots, s\}$, by

$$
\tilde{G}_{J}^{q}=\left\{g \in I(M): g \cdot L_{J}(q)=L_{J}(q)\right\}_{0} .
$$

In the same way as before, one has that $G_{J}^{q}$ is a normal subgroup of $\tilde{G}_{J}^{q}$. Let

$$
\bar{H}_{J}^{q}=\left\{g \in I(M): g \text { acts trivially on } L_{J}(q)\right\}_{0} .
$$

One has that $\bar{H}_{J}^{q}$ is also a normal subgroup of $\tilde{G}_{J}^{q}$. Observe that $G_{i}^{q}$ acts trivially on $L_{J}(q)$, if $i \notin J$. Then, by Lemma 3.5 .

$$
\bar{H}_{J}^{q}=\bar{H}^{q} \times \hat{G}_{J}^{q}
$$

where

$$
\hat{G}_{J}^{q}=\prod_{i \notin J} G_{i}^{q}
$$

One has also that

$$
\tilde{G}_{J}^{q}=G_{J}^{q} \times \bar{H}_{J}^{q} \quad \text { (almost direct product). }
$$

Observe that $I(M)_{q} \subset \tilde{G}_{J}^{q}$, since the full isotropy at $q$ leaves invariant $L_{J}(q)$. Then,

$$
\operatorname{Lie}\left(I(M)_{q}\right)=\mathfrak{k}_{J}^{q} \oplus \overline{\mathfrak{h}}_{J}^{q} \quad \text { (direct sum of ideals), }
$$

where $\overline{\mathfrak{h}}_{J}^{q}=\operatorname{Lie}\left(\bar{H}_{J}^{q}\right)$ and $\mathfrak{k}_{J}^{q}=\bigoplus_{j \in J} \mathfrak{k}_{j}^{q}$.

3.1. The bracket formula. We recall the Koszul formula, which gives the LeviCivita connection in terms of the Riemannian metric tensor and the Lie bracket:

$$
\begin{aligned}
2\left\langle\nabla_{X} Y, Z\right\rangle= & X\langle Y, Z\rangle+Y\langle X, Z\rangle-Z\langle X, Y\rangle \\
& +\langle[X, Y], Z\rangle-\langle[X, Z], Y\rangle-\langle[Y, Z], X\rangle .
\end{aligned}
$$

Assume now that $X, Y, Z$ are Killing fields. Since the flow of a Killing field preserves the metric tensor, then the Lie derivative of the metric along this Killing field is zero. Then

$$
X\langle Y, Z\rangle=\langle[X, Y], Z\rangle+\langle Y,[X, Z]\rangle
$$


and the same if one permutes $X, Y, Z$. By making use of these relations in the Koszul formula one obtains the well known formula for the Levi-Civita connection in terms of Killing fields:

$$
2\left\langle\nabla_{X} Y, Z\right\rangle=\langle[X, Y], Z\rangle+\langle[X, Z], Y\rangle+\langle[Y, Z], X\rangle .
$$

Assume now that $Y$ is parallel at $q$. Then

$$
\langle[X, Y], Z\rangle_{q}+\langle[X, Z], Y\rangle_{q}+\langle[Y, Z], X\rangle_{q}=0 .
$$

Proposition 3.6. Let $M$ be a homogeneous Riemannian manifold and let $\mathcal{L}$ be its foliation of symmetry (i.e., the elements of $\mathcal{L}$ are the integral manifolds of the distribution of symmetry $\mathfrak{s})$. Assume that the metric of $M$ projects down to $M / \mathcal{L}$ (only locally, since $M / \mathcal{L}$ could be not a manifold if the elements of $\mathcal{L}$ are not closed submanifolds). Let $q \in M$ and let $X$ be a Killing field which is parallel at $q$ (observe that $X(q) \in T_{q} L(q)$, where $L(q)$ is the element of $\mathcal{L}$ containing $\left.q\right)$. Let $\xi, \eta$ be Killing fields of $M$ such that their restriction to $L(q)$ is always perpendicular to $L(q)$. Then

$$
\langle[\xi, X], \eta\rangle_{q}=-\frac{1}{2}\langle X,[\xi, \eta]\rangle_{q} .
$$

Proof. In the equality previous to the proposition, rename $Y$ by $X, X$ by $\xi$ and $Z$ by $\eta$. So,

$$
\langle[\xi, X], \eta\rangle_{q}+\langle[\xi, \eta], X\rangle_{q}+\langle[X, \eta], \xi\rangle_{q}=0 .
$$

Now observe that

$$
0=X(q)\langle\xi, \eta\rangle=\left\langle\nabla_{X} \xi, \eta\right\rangle_{q}+\left\langle\xi, \nabla_{X} \eta\right\rangle_{q},
$$

since the metric projects down to the quotient (observe that the Killing fields are projectable since their flow preserve the foliation of symmetry).

Observe that, since $\nabla$ is torsion free and $(\nabla X)_{q}=0$, then $\left(\nabla_{X} \xi\right)_{q}=[X, \xi]_{q}$ and $\left(\nabla_{X} \eta\right)_{q}=[X, \eta]_{q}$. So, from equality 3.6 , one obtains that $\langle[X, \xi], \eta\rangle_{q}+\langle\xi,[X, \eta]\rangle_{q}=$ 0 . Thus, $\langle[X, \eta], \xi\rangle_{q}=\langle[\xi, X], \eta\rangle_{q}$. Therefore, by making use of this equality in 3.5 . one gets the desired formula.

Remark 3.7. Proposition 3.6 remains true is one replace $\mathcal{L}$ by the foliation $\mathcal{L}_{i}$, whose leaves are $L_{i}(x)$. If $i>0$, then the quotient is a manifold since the leaves are compact and hence the orbit of a compact Lie group.

\section{Proof of Theorem A}

Assumption. In the following we will assume that $M=G / H$ is a compact normal homogeneous Riemannian manifold, which is locally irreducible and not locally symmetric ( $G$ compact and connected).

If the isotropy $H$, let us say at $q$, has fixed non-zero vectors on $T_{q} M$, then the full (connected) isometry group $I_{0}(M)$ is in general bigger than $G$. If $G \subsetneq$ $I_{0}(M)$, then the metric on $M$ is not anymore normal homogeneous with respect to the presentation $M=I_{0}(M) /\left(I_{0}(M)\right)_{q}$. Otherwise, the transvection group of the canonical connection would be $I_{0}(M)$. A contradiction (see Section 2).

A transvection $X$ at $q$, i.e. a Killing field which is parallel at $q$, may not lie in $\mathfrak{g}$. In fact, as it will follow from our main result, it will never lie in $\mathfrak{g}$ (unless the fixed vectors of $H$ coincides with $T_{q}\left(G_{0} \cdot q\right)$, where $G_{0}$ is the abelian part of $G$ ). 
Since $M$ is homogeneous, the distribution $x \mapsto \mathfrak{s}_{x}$ is $G$-invariant and thus nonsingular and $C^{\infty}$. In particular, $i_{\mathfrak{s}}(M)=\operatorname{dim}\left(\mathfrak{s}_{q}\right)$. Observe that $\mathfrak{p}^{g \cdot q}=\operatorname{Ad}(g) \mathfrak{p}^{q}$ and $\mathfrak{k}^{g \cdot q}=\operatorname{Ad}(g) \mathfrak{k}^{q}$.

Lemma 4.1. Let $\Sigma(q)$ be the connected component of the fixed points of $H$ in $M$ (or, equivalently, $\Sigma(q)$ is the integral manifold by $q$ of the distribution $\mathcal{D}$ of fixed vectors of the isotropies). Then $\Sigma(q)$ is a local factor of $L(q)$.

Proof. From Reg10, one has that the full connected group of isometries $I_{0}(M)$ of $M$ is $G_{\mathrm{ss}} \times F$, where $G_{\mathrm{ss}}$ is the semisimple part of $G$ and the Killing fields induced by $F$ are the $G$-invariant vector fields (such a field is determined uniquely by a vector in $\mathcal{D}_{q}$ ). Then the flow of any Killing field induced by $F$ preserve the (autoparallel) distribution $\mathcal{D}$ of fixed vectors of the isotropies. Observe that $G_{\mathrm{ss}}$ also preserves $\mathcal{D}$, since $G$ does so. So, $\mathcal{D}$ is preserved by $I_{0}(M)$. In particular, $\left.\mathcal{D}\right|_{L(q)}$ is preserved by the transvection group $G^{q} \subset I_{0}(M)$ of $L(q)$. Then $\left.\mathcal{D}\right|_{L(q)}$ is a parallel distribution of $L(q)$, which contains, by Lemma 4.2, the whole flat factor $L_{0}(q)$. This implies the assertion.

Lemma 4.2. Let $L_{0}(q)=G_{0}(q) \cdot q$ be the flat part of $L(q)$. Then $T_{q}\left(L_{0}(q)\right)$ is included in the set of fixed vectors in $T_{q} M$ of $H$.

Proof. Let $X$ be a Killing field induced by $H$. Then $X$ is bounded, since $M$ is compact. The one-parameter group of isometries induced by $X$ must leave $L_{0}(q)$ invariant. So, $\left.X\right|_{L_{0}(q)}$ is always tangent to $L_{0}(q)$. Since $X$ is bounded and $L_{0}(q)$ is flat, then $\left.X\right|_{L_{0}(q)}$ must be parallel. Since $X(q)=0$, then $\left.X\right|_{L_{0}(q)}=0$. The assertion follows since $H$ is connected.

Lemma 4.3. Let $M=G / H$ be compact locally irreducible normal homogeneous space which is not locally symmetric. Let $L_{i}(q)$ be a de Rham factor by $q$ of $L(q)$ which is perpendicular at $q$ to the factor $\Sigma(q)$. Let $X$ be a transvection at $q$ lying in $\mathfrak{g}_{i}^{q}=\operatorname{Lie}\left(G_{i}^{q}\right)$. Then $X$ lies in $\mathfrak{g}=\operatorname{Lie}(G)$.

Observe that the fact that $L_{i}(q)$ is perpendicular to $\Sigma(q)$ is equivalent, by Lemma 4.1 and Lemma 4.2 to $L_{i}(q)$ not being contained in $\Sigma(q)$. Observe that $i \geq 1$, by the previous lemma.

Proof of Lemma 4.3. We have that $I_{0}(M)=G_{\mathrm{ss}} \times \tilde{\Sigma}$ where $\tilde{\Sigma}$ is $\Sigma(q)$, but regarded as a Lie group: the Killing fields on $M$ induced by $\tilde{\Sigma}(q)$ are the $G$ invariant fields, which are determined by its initial condition, which is a vector in $T_{q}(\Sigma(q)$ ) (see Section 21. Let $\tilde{\Sigma}_{\mathrm{ss}}$ be the semisimple part of the Lie group $\tilde{\Sigma}$. We need the following auxiliary result.

Sublemma. $\tilde{\Sigma}_{\mathrm{ss}} \subset G_{J}^{q}$, where $J=\left\{j \in\{1, \ldots, s\}: L_{j}(q) \subset \Sigma_{\mathrm{Ss}}(q)\right\}$ and $\Sigma_{\mathrm{SS}}(q)$ is the semisimple local factor of the symmetric space $\Sigma(q)$.

Proof. We have that $\tilde{\Sigma}_{\mathrm{SS}}$ is a semisimple normal subgroup of

$$
\tilde{G}_{J}^{q}=G_{J}^{q} \times \bar{H}_{J}^{q} \quad \text { (almost direct product), }
$$

since it leaves $\Sigma_{\mathrm{ss}}(q)$ invariant and it is a normal subgroup of $I(M)$. Let us show that $\tilde{\Sigma}_{\mathrm{ss}} \cap \bar{H}_{J}^{q}$ is discrete. In fact, if $X$ is a Killing field in the Lie algebra of this group, then $X(q)=0$, since $\bar{H}_{J}^{q}$ is contained in the isotropy $(I(M))_{q}$. But a Killing field in the Lie algebra of $\tilde{\Sigma}_{\mathrm{ss}} \subset \tilde{\Sigma}$ is determined by its value at $q$. Then $X=0$ and therefore $\tilde{\Sigma}_{\mathrm{ss}} \subset G_{J}^{q}$ (we are using here that $\tilde{\Sigma}_{\mathrm{ss}}$ has no abelian part). 
We continue with the proof of Lemma 4.3 . By the above sublemma, $\tilde{\Sigma}_{\mathrm{ss}} \subset G_{J}^{q}$. But, since $\left[\mathfrak{g}_{i}^{q}, \operatorname{Lie}\left(G_{J}^{q}\right)\right]=\{0\}$ and $\mathfrak{g}_{i}^{q} \cap \operatorname{Lie}\left(G_{J}^{q}\right)=\{0\}$, one has that $\left[\mathfrak{g}_{i}^{q}, \operatorname{Lie}\left(\Sigma_{\mathrm{ss}}\right)\right]=$ $\{0\}$ and $\mathfrak{g}_{i}^{q} \cap \operatorname{Lie}\left(\Sigma_{\mathrm{ss}}\right)=\{0\}$. This implies that

$$
\mathfrak{g}_{i}^{q} \subset \operatorname{Lie}\left(G_{\mathrm{ss}}\right) \oplus \mathfrak{a} \subset \mathfrak{g} \oplus \mathfrak{a} \oplus \operatorname{Lie}\left(\Sigma_{\mathrm{ss}}\right)=\operatorname{Lie}(I(M))
$$

(direct sum of ideals), where $\mathfrak{a}$ is the abelian Lie algebra associated to the flat part $\tilde{\Sigma}_{0}$ of $\tilde{\Sigma}$. Since $\mathfrak{g}_{i}^{q}$ is semisimple one must have that $\mathfrak{g}_{i}^{q} \subset \operatorname{Lie}\left(G_{\mathrm{ss}}\right) \subset \mathfrak{g}$. This shows the lemma.

Let $X$ be a transvection at $q$ which lies in $\mathfrak{g}_{i}^{q}=\operatorname{Lie}\left(G_{i}^{q}\right)$, where the de Rham factor $L_{i}(q)=G_{i}^{q} \cdot q$ is perpendicular at $q$ to the factor $\Sigma(q)$ of $L(q)$. From Lemma 4.3 we have that $X$ lies in $\mathfrak{g}=\operatorname{Lie}(G)$. Choose $Y \in \mathfrak{m}=\mathfrak{h}^{\perp}$ such that $Y \cdot q=\frac{1}{2} X \cdot q$. Recall that the linear map, from $\mathfrak{m}$ into itself, $U \mapsto[W, U]_{\mathfrak{m}}$ is skew-symmetric for all $W \in \mathfrak{m}$ (since $M=G / H$ has a normal homogeneous metric). Also observe that any $\xi \in \mathfrak{m}$, regarded as the Killing field $x \mapsto \xi \cdot x$, which is perpendicular at $q$ to $L(q)$ remains always perpendicular to $L(q)$ (see Remark 4.9p. Then, for any arbitrary $\xi, \eta \in \mathfrak{m}$ which are perpendicular to $L_{i}(q)$ at $q$,

$$
\langle[\xi, Y], \eta\rangle_{q}=-\langle Y,[\xi, \eta]\rangle_{q} .
$$

Then, from Proposition 3.6 , one obtains that $Z=X-Y$ satisfies

$$
\langle[\xi, Z], \eta\rangle_{q}=0 .
$$

This implies that the Killing field $Z$, regarded in the quotient $M / \mathcal{L}_{i}$ of $M$ by the foliation $\mathcal{L}_{i}$ is identically zero (since its initial conditions are both zero). The quotient $M / \mathcal{L}_{i}$ can be regarded globally, since the leaves $L_{i}(x)$ are compact. Then, the Killing field $Z$ of $M$ is always tangent to the leaves of the foliation $\mathcal{L}_{i}$. Observe that $Z \cdot q$ is an arbitrary vector of $T_{q}\left(L_{i}(q)\right)$, since $Z \cdot q=\frac{1}{2} X \cdot q$. Observe that the same argument shows that there is a Killing field, always tangent to the leaves of the foliation $\mathcal{L}_{i}$, with an arbitrary value in $T_{x}\left(L_{i}(x)\right)$, for any fixed $x \in M$.

Let now $\hat{\mathfrak{g}}_{i}$ be the ideal of $\mathfrak{g}$ which consists of the Killing field that are always tangent to the leaves of $\mathcal{L}_{i}$. Let $\hat{G}_{i}$ be the Lie normal subgroup of $G$ associated to $\hat{\mathfrak{g}}_{i}$. The orbits of $\hat{G}_{i}$, by what previously observed, are the leaves $L_{i}(x)$ of the foliation $\mathcal{L}_{i}$. Let $G_{i}^{\prime}$ be the Lie subgroup of $G$, associated to the complementary ideal $\mathfrak{g}_{i}^{\prime}:=\left(\hat{\mathfrak{g}}_{i}\right)^{\perp}$. We have that

$$
G=\hat{G}_{i} \times G_{i}^{\prime} \quad \text { (almost direct product) }
$$

and that $G_{i}^{\prime}$ acts transitively on the quotient $M / \mathcal{L}_{i}=I_{0}(M) / \tilde{G}_{i}^{q}$. Here, we denote by $\tilde{G}_{i}^{q}$ the group $\tilde{G}_{\{i\}}^{q}$, according with the notation given in 3.1 .

Let now $Z \in \hat{\mathfrak{g}}_{i}$ be such that its associated Killing field on $M$ vanishes identically on $L_{i}(x)$, for some $x \in M$. If $y \in M$, then there exists $g^{\prime} \in G_{i}^{\prime}$ such that $g^{\prime} \cdot L_{i}(x)=L_{i}(y)$, since $G_{i}^{\prime}$ acts transitively on the quotient $M / \mathcal{L}_{i}$. We may assume, by replacing $x$ by another element in $L_{i}(x)$, that $g^{\prime} \cdot x=y$. Then

$$
Z \cdot y=Z \cdot\left(g^{\prime} \cdot x\right)=d m_{g^{\prime}}\left(\operatorname{Ad}\left(\left(g^{\prime}\right)^{-1}\right)(Z)\right) \cdot x=d m_{g^{\prime}}(Z \cdot x)=0,
$$

where $m$ denotes de action of $G$ on $M$. This shows that the Killing field associated to $Z$ vanishes identically. So, $Z=0$.

Lemma 4.4. We have that the normal subgroup $\hat{G}_{i}$ of $G$ is contained in the transvection group $G_{i}^{x}$ of $L_{i}(x)$, for any $x \in M$. 
Proof. If $x \in M$, then

$$
\hat{G}_{i} \subset \tilde{G}_{i}^{x}=G_{i}^{x} \times \bar{H}_{i}^{x}
$$

since $\hat{G}_{i}$ leaves invariant any leaf of $\mathcal{L}_{i}$. We have that $\hat{G}_{i} \cap \bar{H}_{i}^{q}$ is discrete. Otherwise, there would exists a $Z \in \hat{\mathfrak{g}}_{i}$ which vanishes identically on $L_{i}(x)$ and therefore, as previously observed, $Z=0$. The conclusion follows, by making use that $\hat{G}_{i}$ is a normal subgroup of $\tilde{G}_{i}^{x}$ and the fact that $G_{i}^{x}$ is semisimple.

Proposition 4.5. The locally symmetric (irreducible) space $L_{i}(x)$ is of group type. Namely, $G_{i}^{x}=K \times K$ (almost direct product), where $K$ is a simple Lie group (of compact type). Moreover, $\hat{G}_{i}$ coincides with one of the factors $K$.

Proof. Assume first that $\hat{G}_{i}=G_{i}^{x}$. Then any transvection $X \in \mathfrak{p}_{i}^{x} \subset \mathfrak{g}_{i}^{x}$ at $x$ lies in $\hat{\mathfrak{g}}_{i}$. Let now $X_{1}, \ldots, X_{r} \in \mathfrak{p}_{i}^{x}$ (i.e. transvections at $x$ ) be such that $X_{1} \cdot x, \ldots, X_{r} \cdot x$ is a basis of $T_{x}\left(L_{i}(x)\right)$. Then $X_{1}, \ldots, X_{r}$ is a local trivialization of the distribution $\mathfrak{s}_{i}$ of tangent spaces to the leaves of $\mathcal{L}_{i}$. Since $X_{1}, \ldots, X_{r}$ are parallel at $x$ we conclude that the distribution $\mathfrak{s}_{i}$ is parallel at $x$. Since $x \in M$ is arbitrary we have that the (non-trivial) distribution $\mathfrak{s}_{i}$ is parallel and so $M$ locally splits since $\operatorname{dim} \mathfrak{s}_{i}<n=\operatorname{dim} M$ because $M$ is not symmetric. A contradiction.

So, $\hat{G}_{i}$ is a proper, non-trivial, normal subgroup of $G_{i}^{x}$. Since $L_{i}(x)$ is a locally irreducible symmetric space the isometry group $G_{i}^{x}$ is semi-simple. But it cannot be simple, since it has a proper normal subgroup. Then $L_{i}(x)$ must be of group type and $G_{i}^{x}=K \times K$ (almost direct product), with $K$ simple, since $L_{i}(x)$ is locally irreducible.

Remark 4.6. Recall that $L_{i}(x)$ is Lie group with a bi-invariant metric (since it is a globally symmetric space of the group type).

Remark 4.7. Let us fix $q \in M$. Let $\Psi: \hat{\mathfrak{g}}_{i} \rightarrow \mathfrak{g}_{i}^{\prime}$ be defined as follows. Given $X \in \hat{\mathfrak{g}}_{i}, \Psi(X)$ is the unique element of $\mathfrak{g}_{i}^{\prime}$ such that $\Psi(X) \cdot q=-X \cdot q$. We get that $\Psi: \hat{\mathfrak{g}}_{i} \rightarrow \Psi\left(\hat{\mathfrak{g}}_{i}\right)$ is an isomorphism of Lie algebras. Moreover, $\mathfrak{g}_{i}^{q}=\hat{\mathfrak{g}}_{i} \oplus \Psi\left(\hat{\mathfrak{g}}_{i}\right)$ and

$$
\mathfrak{k}_{i}^{q}=\left\{X+\Psi(X): X \in \hat{\mathfrak{g}}_{i}\right\}=: \operatorname{diag}\left(\hat{\mathfrak{g}}_{i} \oplus \Psi\left(\hat{\mathfrak{g}}_{i}\right)\right) .
$$

Let $\mathfrak{h}^{\prime}$ be the ideal of $\mathfrak{h}=\operatorname{Lie}(H) \subset \mathfrak{g}$ which consists of the Killing fields that vanish identically on the leaf $L_{i}(q)$. We have, from Lemma 4.3 , that $\mathfrak{k}_{i}^{q} \subset \mathfrak{h}$. Then with the same arguments as in 3.3 . for the case where $\mathfrak{h}$ is the full isotropy algebra, we can decompose

$$
\mathfrak{h}=\mathfrak{h}^{\prime} \oplus \mathfrak{k}_{i}^{q} \quad \text { (direct sum of ideals). }
$$

Since $\mathfrak{k}_{i}^{q}$ is simple, from Remark 2.1 the above decomposition must be orthogonal with respect to the $\operatorname{Ad}(G)$-invariant inner product $\langle\cdot, \cdot\rangle$ on $\mathfrak{g}$ (or, more generally, with respect to any $\operatorname{Ad}(G)$-invariant symmetric bilinear form on $G$ ).

Since $\mathfrak{h}^{\prime} \subset \overline{\mathfrak{h}}_{i}^{q}$ and $\overline{\mathfrak{h}}_{i}^{q}$ is an ideal of $\tilde{\mathfrak{g}}_{i}^{q}$, we get that $\mathfrak{h}^{\prime}$ is an ideal of $\mathfrak{h}^{\prime} \oplus \mathfrak{g}_{i}^{q}$. This decomposition, since $\mathfrak{g}_{i}^{q}$ is semisimple, must be orthogonal by Remark 2.1.

On the other hand, the decomposition $\mathfrak{g}=\mathfrak{h}^{\prime} \oplus \mathfrak{k}_{i}^{g} \oplus \mathfrak{m}$ is also orthogonal. So, we get that $\left(\mathfrak{k}_{i}^{q}\right)^{\perp} \cap \mathfrak{g}_{i}^{q} \subset \mathfrak{m}$.

Let us define

$$
\mathfrak{m}_{1}:=\left(\mathfrak{k}_{i}^{q}\right)^{\perp} \cap \mathfrak{g}_{i}^{q} \subset \mathfrak{m} \quad \text { and } \quad \mathfrak{m}_{2}:=\left(\mathfrak{m}_{1}\right)^{\perp} \cap \mathfrak{m} .
$$

Keeping the notation of Proposition 4.5 and Remark 4.6 we have that the decomposition $\mathfrak{g}_{i}^{q}=\hat{\mathfrak{g}}_{i} \oplus \hat{\mathfrak{g}}_{i}$, where the first summand is regarded as an ideal of $\mathfrak{g}$ and the second one as a subalgebra of $\mathfrak{g}_{i}^{\prime}$, must be orthogonal (see Remark 2.1). 
Moreover, just by taking a positive multiple of the $\operatorname{Ad}(G)$-invariant inner product $\langle\cdot, \cdot\rangle$ on $\mathfrak{g}$, we may assume that the restriction of $\langle\cdot, \cdot\rangle$ to $\mathfrak{g}_{i}^{q}=\hat{\mathfrak{g}}_{i} \oplus \hat{\mathfrak{g}}_{i}$ has the form

$$
\left.\langle\cdot, \cdot\rangle\right|_{\mathfrak{g}_{i}^{q}}=B \oplus \lambda B
$$

for some $\lambda>0$, where $B$ is the negative of the Killing form of $\hat{\mathfrak{g}}_{i}$.

With these notation and identifications we have

$$
\mathfrak{k}_{i}^{q}=\left\{(v, v): v \in \hat{\mathfrak{g}}_{i}\right\}, \quad \mathfrak{p}_{i}^{q}=\left\{\left(\frac{1}{2} v,-\frac{1}{2} v\right): v \in \hat{\mathfrak{g}}_{i}\right\}
$$

and

$$
\mathfrak{m}_{1}=\left\{\left(\frac{-\lambda}{1+\lambda} v, \frac{1}{1+\lambda} v\right): v \in \hat{\mathfrak{g}}_{i}\right\} .
$$

With this notation, if $X=(u, v) \in \mathfrak{g}_{i}^{q}$, then

$$
X \cdot q=(u, v) \cdot q=(u-v) \cdot q .
$$

Let $a=\frac{1}{2}(\lambda-1)$. Then, for all $v \in \hat{\mathfrak{g}}_{i}$,

$$
\left(\left(\frac{1}{2}+a\right) v,-\frac{1}{2} v\right) \in \mathfrak{m}_{1}
$$

In fact, if $(w, w) \in \mathfrak{k}_{i}^{q}$, then

$$
\begin{aligned}
\left\langle(w, w),\left(\left(\frac{1}{2}+a\right) v,-\frac{1}{2} v\right)\right\rangle & =\left\langle w,\left(\frac{1}{2}+a\right) v\right\rangle+\lambda\left\langle w,-\frac{1}{2} v\right\rangle \\
& =\left(\frac{1}{2}+a-\frac{\lambda}{2}\right)\langle w, v\rangle \\
& =\left(\frac{1}{2}+\frac{1}{2}(\lambda-1)-\frac{\lambda}{2}\right)\langle w, v\rangle=0 .
\end{aligned}
$$

Note that

$$
\left(\left(\frac{1}{2}+a\right) v,-\frac{1}{2} v\right) \cdot q=(1+a) v \cdot q
$$

Observe, identifying $T_{q} M \simeq \mathfrak{m}$, that

$$
\mathfrak{m}_{2}=\left(T_{q}\left(L_{i}(q)\right)\right)^{\perp} .
$$

Let now $\xi, \eta \in \mathfrak{m}_{2}$. As we have previously observed, the Killing fields on $M$ induced by $\xi, \eta$ are always perpendicular to $L_{i}(q)$. If $X=\left(\frac{1}{2} v,-\frac{1}{2} v\right) \in \mathfrak{p}_{i}^{q}$ is a transvection at $q$, then, by Proposition 3.6 .

$$
\langle[\xi, X], \eta\rangle_{q}=-\frac{1}{2}\langle X,[\xi, \eta]\rangle_{q} .
$$

Since $\hat{\mathfrak{g}}_{i}$ is an ideal,

$$
\langle[\xi,(a v, 0)], \eta\rangle_{q}=0 .
$$

Then, if $Y=X+(a v, 0)$,

$$
\langle[\xi, Y], \eta\rangle_{q}=\langle[\xi, X], \eta\rangle_{q}=-\frac{1}{2}\langle X,[\xi, \eta]\rangle_{q}=-\frac{1}{2}\langle X \cdot q,[\xi, \eta] \cdot q\rangle .
$$

Since $X \cdot q=\frac{1}{1+a} Y \cdot q$, the above equality yields

$$
\langle[\xi, Y], \eta\rangle_{q}=-\frac{1}{2(1+a)}\langle Y \cdot q,[\xi, \eta] \cdot q\rangle
$$


But on the other hand, since $Y \in \mathfrak{m}_{1} \subset \mathfrak{m}$, one must have that

$$
\langle[\xi, Y], \eta\rangle_{q}=-\langle Y \cdot q,[\xi, \eta] \cdot q\rangle .
$$

Then, either $\frac{1}{2(1+a)}=1$, or $\mathfrak{m}_{1}$ is perpendicular to $\left[\mathfrak{m}_{2}, \mathfrak{m}_{2}\right]_{\mathfrak{m}}$. In the first case

$$
1=\frac{1}{2\left(1+\frac{1}{2}(\lambda-1)\right)}=\frac{1}{1+\lambda}
$$

A contradiction, since $\lambda>0$. In the second case the distribution $\left(\mathfrak{s}_{i}\right)^{\perp}$ is integrable and hence totally geodesic. Also a contradiction (see Remark 4.8).

Then there does not exist a transvection at $q$ which is perpendicular at $q$ to the vectors fixed by the isotropy. This proves Theorem A.

Remark 4.8. We denote by $\tilde{\xi}$ the Killing field on $M$ induced by $\xi$, which is given by $\tilde{\xi}_{x}=\xi \cdot x$ (and the same for $\eta$ ). We have that $[\xi, \eta]_{\mathfrak{m}} \in \mathfrak{m}_{2}$, and we want to see that $[\tilde{\xi}, \tilde{\eta}]$ lies in the distribution $\left(\mathfrak{s}_{i}\right)^{\perp}$. Recall that if $q^{\prime}=g \cdot q$ and $\bar{\xi}, \bar{\eta}$ are the rightinvariant fields on $G$ with $\bar{\xi}_{e}=\xi_{e}$ and $\bar{\eta}_{e}=\eta_{e}$, then $\tilde{\xi}_{q^{\prime}}=d \pi\left(\bar{\xi}_{g}\right), \tilde{\eta}_{q^{\prime}}=d \pi\left(\bar{\eta}_{g}\right)$ and $[\tilde{\xi}, \tilde{\eta}]_{q^{\prime}}=d \pi\left([\bar{\xi}, \bar{\eta}]_{g}\right)$, since $[\bar{\xi}, \bar{\eta}]$ is right-invariant (here $\pi: G \rightarrow M$ is the projection map).

On the other hand, we may identify $T_{q^{\prime}} M$ with $\operatorname{Ad}\left(g^{-1} \mathfrak{m}\right)$ via $Z \mapsto Z \cdot q^{\prime}$. Note that the isotropy at $q^{\prime}$ is $\operatorname{Ad}\left(g^{-1}\right) \mathfrak{h}$ and $\left(\mathfrak{s}_{i}\right) \frac{\perp}{q^{\prime}}=\left(\operatorname{Ad}\left(g^{-1}\right) \mathfrak{m}_{2}\right) \cdot q^{\prime}$. So,

$$
\begin{aligned}
{[\tilde{\xi}, \tilde{\eta}]_{q^{\prime}} } & =d \pi\left([\bar{\xi}, \bar{\eta}]_{g}\right)=d \pi\left(d r_{g}[\bar{\xi}, \bar{\eta}]_{e}\right) \\
& =-d \pi\left(d r_{g}[\xi, \eta]_{e}\right)=-d \pi\left(d l_{g} \operatorname{Ad}\left(g^{-1}\right)[\xi, \eta]_{e}\right) \\
& =-d l_{g} d \pi\left(\operatorname{Ad}\left(g^{-1}\right)[\xi, \eta]_{e}\right) \in\left(\mathfrak{s}_{i}\right)_{q^{\prime}}^{\perp},
\end{aligned}
$$

where we denote by $l_{g}$ the left-multiplication in $G$ and $M$. Therefore, $\left(\mathfrak{s}_{i}\right)^{\perp}$ is integrable and autoparallel.

Remark 4.9. Let $\xi \in \mathfrak{m}$ such that the Killing field $\tilde{\xi}$ defined by $\tilde{\xi}_{x}=\xi \cdot x$ is perpendicular to $L(q)$ at $q$. Recall that $T_{x} L(q)=\mathfrak{s}_{x}$ for all $x \in L(q)$. Let $\mathfrak{m}_{0} \subset \mathfrak{m}$ be the subspace such that $\mathfrak{s}_{q}=\mathfrak{m}_{0} \cdot q$. Since the distribution of symmetry is $G$ invariant, we have that $\mathfrak{s}_{q^{\prime}}=\left(\operatorname{Ad}\left(\mathfrak{m}_{0}\right)\right) \cdot q^{\prime}$, where $g \in G$ and $q^{\prime}=g \cdot q$. Now, $\tilde{\xi}_{q^{\prime}}=(\operatorname{Ad}(g) \xi) \cdot q^{\prime}$ is perpendicular to $\mathfrak{s}_{q^{\prime}}$. Since $g$ is arbitrary, we conclude that if $\tilde{\xi}$ is perpendicular to $L(q)$ at $q$, it is always perpendicular to $L(q)$.

Example 4.10 (Stiefel manifolds). Let us consider the Stiefel manifold $M=$ $\mathrm{SO}(n+k) / \mathrm{SO}(n)$, with $n, k \geq 2$, endowed with the normal homogeneous metric. It is well known, as it follows from the Serre long exact sequence of homotopies, that $M$ is simply connected. Moreover, $M$ is an irreducible Riemannian manifold (see Remark 4.11). Then $M$ has index of symmetry $i_{\mathfrak{s}}(M)=\frac{1}{2} k(k-1)$. Moreover, the (connected) set of fixed points of $\mathrm{SO}(n)$ in $M$, which contains the orthogonal $k$-frame $B=\left(e_{1}, \ldots, e_{k}\right)$, is isomorphic to $\mathrm{SO}(k)$. Here we consider the standard inclusions $\mathrm{SO}(n) \simeq\left(\begin{array}{cc}I_{k} & 0 \\ 0 & \mathrm{SO}(n)\end{array}\right)$ and $\mathrm{SO}(k) \simeq\left(\begin{array}{cc}\mathrm{SO}(k) & 0 \\ 0 & I_{n}\end{array}\right)$ inside $\mathrm{SO}(n+k)$. Thus, the leaf of symmetry $L(B)$ is the symmetric space of the group type $\mathrm{SO}(k) \simeq(\mathrm{SO}(k) \times \mathrm{SO}(k)) / \mathrm{SO}(k)$.

Remark 4.11. Let $M=G / H$ be a simply connected naturally reductive Riemannian manifold, where $G$ is the group of transvections of the canonical connection. If $M$ is a normal homogeneous space, $G$ must always coincides with the group of transvections, provided $G$ acts effectively. Let $M=M_{0} \times M_{1} \times \cdots \times M_{r}$ be the de 
Rham decomposition ( $M_{0}$ is, eventually, trivial). Then $G=G_{0} \times G_{1} \times \cdots \times G_{r}$ and $H=H_{0} \times H_{1} \times \cdots \times H_{r}$, where $H_{i} \subset G_{i}$ and $M_{i}=G_{i} / H_{i}$ for all $i=0,1, \ldots, r$. It is not easy to find this decomposition through the mathematical literature. We found such a fact in [AleEM], but without proof. So, we next give the argument for such a decomposition.

Let $p=\left(p_{0}, \ldots, p_{r}\right)=e H \in G / H$ and let $\mathfrak{g}=\mathfrak{m} \oplus \mathfrak{h}$ be the naturally reductive decomposition associated to the naturally reductive metric on $M$. Observe, as it is well known, since $G$ is the group of transvections, that $\mathfrak{g}=\mathfrak{m}+[\mathfrak{m}, \mathfrak{m}]$.

Identifying $T_{p} M \simeq \mathfrak{m}$, let $\mathfrak{m}=\mathfrak{m}_{0} \oplus \mathfrak{m}_{1} \oplus \cdots \oplus \mathfrak{m}_{r}$, where $\mathfrak{m}_{i}=T_{p_{i}} M_{i}$ and $T_{p} M=T_{p_{0}} M_{0} \oplus T_{p_{1}} M_{1} \oplus \cdots \oplus T_{p_{r}} M_{r}$.

If $Z \in \mathfrak{g}$, let $\hat{Z}$ be its associated Killing field $q \mapsto Z \cdot q$. It is well known that $(\nabla \hat{Z})_{p}$ lies in the normalizer of the holonomy algebra at $p$. So, $(\nabla \hat{Z})_{p}$ leaves invariant the tangent space at $p$ of any de Rham factor. Namely, for all $i=0,1, \ldots, r$,

$$
\left(\nabla_{\mathfrak{m}_{i}} \hat{Z}\right)_{p} \subset \mathfrak{m}_{i} .
$$

If $X \in \mathfrak{m}$, then

$$
(\nabla \hat{X})_{p}=D_{X}
$$

where $D=\nabla-\nabla^{c}$ is the difference tensor between the Levi-Civita connection and the canonical connection. Moreover, $\left\langle D_{X} Y, Z\right\rangle$ is a 3 -form. From 4.1 one has that, if $i \neq j$,

$$
D_{\mathfrak{m}_{i}} \mathfrak{m}_{j}=\{0\} .
$$

Let now $X_{i} \in \mathfrak{m}_{i}$, then $d l_{\operatorname{Exp}\left(t X_{i}\right)}$ gives the $\nabla^{c}$-parallel transport along the geodesic $\operatorname{Exp}\left(t X_{i}\right) \cdot p \in M_{i}$. If $u \in\left(\mathfrak{m}_{i}\right)^{\perp}$, then from $4.2 d l_{\operatorname{Exp}\left(t X_{i}\right)} u$ is also parallel with respect to the Levi-Civita connection, along $\operatorname{Exp}\left(t X_{i}\right) \cdot p$.

This implies that $l_{\operatorname{Exp}\left(t X_{i}\right)}$ acts trivially on any other de Rham factor $M_{j}, j \neq i$.

Then, if we define $G_{i}$ to be the Lie subgroup of $G$ whose Lie algebra is generated by $\mathfrak{m}_{i}$ and $H_{i}=\left(G_{i}\right)_{p}$ we obtain the desired decomposition.

\section{The naturally Reductive Case}

In this section we assume that $M=G / H$ is a compact naturally reductive space, with reductive decomposition $\mathfrak{g}=\mathfrak{h} \oplus \mathfrak{m}$. We also assume that $M$ is locally irreducible and non-locally symmetric, and the presentation $G / H$ is given by the transvection group of the canonical connection of $M$. That is to say, $\mathfrak{g}=\mathfrak{m}+[\mathfrak{m}, \mathfrak{m}]$ (which is not, in general, a direct sum). From a well known result due to Kostant Kos56, there exists an $\operatorname{Ad}(G)$-invariant non-degenerate bilinear form $Q$ on $\mathfrak{g}$ such that

$$
Q(\mathfrak{h}, \mathfrak{m})=0,\left.\quad Q\right|_{\mathfrak{m}}=\langle\cdot, \cdot\rangle,
$$

where we denote by $\langle\cdot, \cdot\rangle$ the Riemannian metric at $T_{e H} M \simeq \mathfrak{m}$. In particular, it follows that $Q$ is non-degenerate when restricted to $\mathfrak{h}$.

We keep the notation from the previous section. More precisely, if $L(q)$ is the leaf of symmetry by $q \in M$, the de Rham decomposition of $L(q)$ is given by

$$
L(q)=L_{0}(q) \times L_{1}(q) \times \cdots \times L_{s}(q),
$$

and the leaf of fixed point of the isotropy $\Sigma(q)$ by $q$ is a local factor of $L(q)$. That is, there exist a subset $J \subset\{0,1, \ldots, s\}$, which must contain 0 , such that

$$
\Sigma(q)=L_{J}(q)=\prod_{j \in J} L_{j}(q) .
$$


If $i \notin J$, we proved that $L_{i}(q)$ is a symmetric space of the group type. Moreover, the ideal $\hat{\mathfrak{g}}_{i} \subset \mathfrak{g}$, which consist of the Killing fields which are always tangent to the foliation with leaves $L_{i}(q), x \in M$, is a simple ideal of the Lie algebra of geometric transvections $\mathfrak{g}_{i}^{q}=\mathfrak{k}_{i}^{q} \oplus \mathfrak{p}_{i}^{q}$. In particular, there exists an ideal $\mathfrak{k}$ contained in the $Q$ orthogonal ideal to $\hat{\mathfrak{g}}_{i}$, which turns out isomorphic to $\hat{\mathfrak{g}}_{i}$, such that $\mathfrak{k}_{i}^{q} \simeq \operatorname{diag}\left(\hat{\mathfrak{g}}_{i} \oplus \hat{\mathfrak{g}}_{i}\right)$.

Recall that $\left.Q\right|_{\mathfrak{g}_{i}^{q}}$ has the form $\lambda B \oplus \mu B$, where $B$ is the negative of the Killing form of $\hat{\mathfrak{g}}_{i}$ and we identify $\mathfrak{g}_{i}^{q} \simeq \hat{\mathfrak{g}}_{i} \oplus \hat{\mathfrak{g}}_{i}$. We have that $\lambda$ and $\mu$ are both nonzero, since $\left.Q\right|_{\mathfrak{h}}$ is non-degenerate.

If $Q$ is not positive defined, then $\lambda, \mu$ cannot have both the same sign. If $\lambda>$ 0 , we get a contradiction with the same argument that we use for the normal homogeneous case (because, we only use that $\mu \neq 0$ ). Therefore, it only remains the case $\lambda<0<\mu$. By rescaling the Riemannian metric on $M$, we may assume that

$$
\left.Q\right|_{\mathfrak{g}_{i}^{q}}=-\lambda B \oplus B, \quad \text { for some } \lambda>0 .
$$

Recall that $\lambda \neq 1$, since $Q$ is non-degenerate on the isotropy $\mathfrak{h}$. Let, $\mathfrak{m}_{1} \subset \mathfrak{m}$ the subspace such that $\mathfrak{m}_{1} \cdot q=T_{q}\left(L_{i}(q)\right)$. So, with our identifications, we have that

$$
\mathfrak{k}_{i}^{q}=\left\{(v, v): v \in \hat{\mathfrak{g}}_{i}\right\}, \quad \mathfrak{p}_{i}^{q}=\left\{\left(\frac{1}{2} v,-\frac{1}{2} v\right): v \in \hat{\mathfrak{g}}_{i}\right\}
$$

and

$$
\mathfrak{m}_{1}=\left\{\left(\frac{1}{1-\lambda} v, \frac{\lambda}{1-\lambda} v\right): v \in \hat{\mathfrak{g}}_{i}\right\} .
$$

In this case, we have that if $a=-\frac{1}{2}(\lambda+1)$, then

$$
\left(\frac{1}{2} v,-\frac{1}{2} v\right)+(a v, 0) \in \mathfrak{m}_{1}
$$

for all $v \in \hat{\mathfrak{g}}_{i}$. Just by following the calculations from the last part of the previous section we get the contradiction

$$
1=\frac{1}{2(a+1)}=\frac{1}{1-\lambda} .
$$

This proves Theorem $\mathrm{B}$.

\section{EXAmples With Distribution OF SYMMETRY NOT OF A GROUP-TYPE}

For a compact (simply connected) naturally reductive space $M=G / H$ the leaves of the symmetric foliation, are always globally symmetric spaces of group type. In fact, if $G$ is the group of transvections of the canonical connection then the symmetric submanifold $S$ by $p=[e]$ coincides with the connected component of the fixed points of $H$ in $M$.

The full isometry group is given by (see Reg10, OR12a)

$$
I_{0}(M)=G_{\mathrm{ss}} \times \tilde{S},
$$

where $\tilde{S}$ is the group of isometries which corresponds to the $G$-invariant vector fields of $M$, and $G_{\mathrm{ss}}$ is the semisimple part of the reductive group $G$. Moreover, the leaves of the symmetric foliation are given by the orbits on $M$ of the group $\tilde{S}$. This implies that the holonomy group $\Phi$ of a fixed leaf $S$, of the symmetric foliation, commutes with $\tilde{S}$. It is not hard to see that $\Phi$ must be isomorphic to $\tilde{S}$ and that $I_{0}(S)=\Phi \times S \simeq S \times S$. 
In the next we will construct examples of compact (simply connected) homogeneous spaces whose foliation of symmetry is not of group type.

Let $G$ be a compact Lie group and let $G^{\prime}, K^{\prime}$ be compact subgroups such that $G \supset G^{\prime} \supset K^{\prime}$. Assume, furthermore, that $G^{\prime}$ is simple and that $\left(G^{\prime}, K^{\prime}\right)$ is a symmetric pair. Observe that $\left(G^{\prime}, K^{\prime}\right)$ is not of group-type, since $G^{\prime}$ is simple.

Let $(\cdot, \cdot)$ be an $\operatorname{Ad}(G)$-invariant inner product in the Lie algebra $\mathfrak{g}$ of $G$. Let

$$
\mathfrak{g}^{\prime}=\mathfrak{k}^{\prime} \oplus \mathfrak{p}^{\prime}
$$

be the Cartan decomposition associated to $\left(G^{\prime}, K^{\prime}\right)$. Since $G^{\prime}$ is simple, then the restriction of $(\cdot, \cdot)$ to $\mathfrak{g}^{\prime}$ is a multiple of the Killing form. So, $\mathfrak{k}^{\prime}$ must be perpendicular to $\mathfrak{p}^{\prime}$ with respect to $\langle\cdot, \cdot\rangle$ (since both subspaces are perpendicular with respect to the Killing form of $\left.\mathfrak{g}^{\prime}\right)$. This is in general false if $G^{\prime}$ is not simple (e.g., if $\left(G^{\prime}, K^{\prime}\right)$ is of group type).

Let now

$$
\mathfrak{m}:=\left(\mathfrak{k}^{\prime}\right)^{\perp}
$$

be the orthogonal complement in $\mathfrak{g}$ with respect to $(\cdot, \cdot)$.

As previously observed, $\mathfrak{p}^{\prime} \subset \mathfrak{m}$. So, if $\mathfrak{m}^{\prime}=\left(\mathfrak{p}^{\prime}\right)^{\perp} \cap \mathfrak{m}$, then

$$
\mathfrak{m}=\mathfrak{m}^{\prime} \oplus \mathfrak{p}^{\prime} \quad \text { (orthogonally). }
$$

Let $\langle\cdot, \cdot\rangle$ be the inner product on $\mathfrak{m}$ defined as follows by the following properties:

a) $\left\langle\mathfrak{k}^{\prime}, \mathfrak{p}^{\prime}\right\rangle=0$

b) the restrictions to $\mathfrak{m}^{\prime}$ of $(\cdot, \cdot)$ and $\langle\cdot, \cdot\rangle$ coincide;

c) $\langle\cdot, \cdot\rangle=2(\cdot, \cdot)$, restricted to the subspace $\mathfrak{p}^{\prime}$.

Let $M:=G / K^{\prime}$ endowed with the $G$-invariant Riemannian metric that at $p=[e]$ coincides with the inner product $\langle\cdot, \cdot\rangle$ of $T_{p} M \simeq \mathfrak{m}$. We will also denote by $\langle\cdot, \cdot \cdot\rangle$ this Riemannian metric. The associated Levi-Civita connection will be denoted by $\nabla$.

Notation. If $X \in \mathfrak{g}$ then $\tilde{X}$ denotes the Killing field of $M$ induced by $X$. Namely, $\tilde{X}(q)=\left.\frac{d}{d t}\right|_{0} \operatorname{Exp}(t X) \cdot q$.

Recall, the well known fact that

$$
[\tilde{X}, \tilde{Y}]=-\widetilde{[X, Y]} .
$$

Lemma 6.1. If $X \in \mathfrak{p}^{\prime}$ then $(\nabla \tilde{X})_{p}=0$.

Proof. Let us first show that $G^{\prime} / K^{\prime}$ is a totally geodesic submanifold of $G / K^{\prime}$. Observe that $\mathfrak{m}^{\prime}$ is the orthogonal complement, with respect to $(\cdot, \cdot)$, of $\mathfrak{g}^{\prime}$ in $\mathfrak{g}$. Then $\mathfrak{m}^{\prime}$ must be $\operatorname{Ad}\left(G^{\prime}\right)$-invariant, since $\mathfrak{g}^{\prime}$ is so.

Let $\xi \in \mathfrak{m}^{\prime}$ and $g^{\prime} \in G^{\prime}$. Since $\mathfrak{g}^{\prime}$ is $G^{\prime}$-invariant,

$$
(\tilde{\xi})_{g^{\prime} K^{\prime}}=d l_{g^{\prime}} \operatorname{Ad}\left(\left(g^{\prime}\right)^{-1}\right) \xi .
$$

Now observe that $\operatorname{Ad}\left(\left(g^{\prime}\right)^{-1}\right) \xi$ belongs to $\mathfrak{m}^{\prime}$ and so it is perpendicular to $G^{\prime} / K^{\prime}$ at $e K^{\prime}$. Since $l_{g^{\prime}}$ is an isometry that preserves $G^{\prime} / K^{\prime}$ one concludes that $(\tilde{\xi})_{g^{\prime} K^{\prime}}$ is perpendicular to $G^{\prime} / K^{\prime}$ at $g^{\prime} K^{\prime}$. Therefore, the Killing field $\tilde{\xi}$, restricted to $G^{\prime} / K^{\prime}$ is always perpendicular to this submanifold. If $A$ is the shape operator of $G^{\prime} / K^{\prime}$ then, for any $U, V$ vector fields on $G^{\prime} / K^{\prime}$,

$$
\left\langle A_{\tilde{\xi}} U, V\right\rangle=-\left\langle\nabla_{U} \tilde{\xi}, V\right\rangle .
$$


But the left hand of the above equality is symmetric on $U, V$ and the right hand is skew (by the Killing equation). Then

$$
A_{\tilde{\xi}}=0=\left\langle\nabla_{U} \tilde{\xi}, V\right\rangle .
$$

Since $\xi$ is any arbitrary normal direction at $p$, we have that $G^{\prime} / K^{\prime}$ is a totally geodesic submanifold of $M$.

Since $X \in \mathfrak{p}^{\prime}$ then $\left.\tilde{X}\right|_{G^{\prime} / K^{\prime}}$ is parallel at $p$, regarded as a Killing field of $G^{\prime} / K^{\prime}$. Then, since $G^{\prime} / K^{\prime}$ is totally geodesic,

$$
\nabla_{Y} \tilde{X}=0
$$

for all $Y \in \mathfrak{p}^{\prime} \simeq T_{p}\left(G^{\prime} / K^{\prime}\right)$. Observe, from last equality and the Killing equation, that

$$
\left\langle\nabla_{\xi} \tilde{X}, Y\right\rangle=0
$$

for any $\xi \in \mathfrak{m}^{\prime}, Y \in \mathfrak{p}^{\prime}$.

Let $\xi, \eta \in \mathfrak{m}^{\prime}$. Then, from equation 3.4 .

$$
\begin{aligned}
2\left\langle\nabla_{\xi} \tilde{X}, \eta\right\rangle & =\left\langle[\tilde{\xi}, \tilde{X}]_{p}, \eta\right\rangle+\left\langle[\tilde{\xi}, \tilde{\eta}]_{p}, X\right\rangle+\left\langle[\tilde{X}, \tilde{\eta}]_{p}, \xi\right\rangle \\
& =-\langle[\xi, X], \eta\rangle-\langle[\xi, \eta], X\rangle-\langle[X, \eta], \xi\rangle \\
& =-([\xi, X], \eta)-2([\xi, \eta], X)-([X, \eta], \xi) \\
& =(X,[\xi, \eta])-2([\xi, \eta], X)+(X,[\xi, \eta])=0,
\end{aligned}
$$

where $(\cdot, \cdot)$ is the $\operatorname{Ad}(G)$-invariant inner product of $\mathfrak{g}$.

From 6.16 .2 and 6.3 one has that $(\nabla \tilde{X})_{p}=0$.

Let $\tilde{\mathfrak{p}}^{\prime}$ denote the $G$-invariant distribution on $M$ with $\tilde{\mathfrak{p}}_{p}^{\prime}=\mathfrak{p}^{\prime}$. Then the distribution $\tilde{\mathfrak{p}}^{\prime}$ is integrable with totally geodesic leaves. In fact, if $g \in G$, then the leaf by $g \cdot p$ of $\tilde{\mathfrak{p}}^{\prime}$ is $g \cdot\left(G^{\prime} / K^{\prime}\right)$ (see the beginning of the proof of Lemma 6.1, where it is proved that $G^{\prime} / K^{\prime}$ is a totally geodesic submanifold of $M$ ).

Lemma 6.2. We keep the notation and general assumptions of this section. Assume, furthermore, that $\left(G, G^{\prime}\right)$ is an irreducible (almost effective) symmetric pair and that $\left(G / K^{\prime},\langle\cdot, \cdot\rangle\right)$ is not a symmetric space. Then the distribution of symmetry of $\left(G / K^{\prime},\langle\cdot, \cdot\rangle\right)$ is exactly $\mathfrak{s}=\tilde{\mathfrak{p}}^{\prime}$ (whose integral manifolds are $g \cdot\left(G^{\prime} / K^{\prime}\right), g \in G$ ).

Proof. We have seen that $\tilde{\mathfrak{p}}^{\prime} \subset \mathfrak{s}$. Then the distribution $\mathfrak{s}$ of $M=G / K^{\prime}$ descends to a distribution $\overline{\mathfrak{s}}$ of the symmetric space $\bar{M}=G / G^{\prime}$. Moreover, $\overline{\mathfrak{s}}$ is $G$-invariant, since $\mathfrak{s}$ is so (because isometries preserve the distribution of symmetry). Then $\overline{\mathfrak{s}}=0$, or $\overline{\mathfrak{s}}=T \bar{M}$. In the first case we have that $\mathfrak{s}=\tilde{\mathfrak{p}}^{\prime}$, as we wanted to prove. In the second case we obtain that $\mathfrak{s}=T M$ and hence $M$ would be symmetric.

We have not found a general argument for deciding when $\left(G / K^{\prime},\langle\cdot, \cdot\rangle\right)$ is not locally symmetric.

6.1. Explicit examples. We keep the notation and assumptions of this section.

Example 6.3 (The unit tangent bundle of the sphere of curvature 2). Set, for $n \geq 1$,

$$
G=\mathrm{SO}(n+1), \quad G^{\prime}=\mathrm{SO}(n), \quad K^{\prime}=\mathrm{SO}(n-1) .
$$

Let $M=G / K^{\prime}$ endowed with the $\mathrm{SO}(n+1)$-invariant metric $\langle\cdot, \cdot\rangle$. Though, for $n=4, \mathrm{SO}(n)$ is not simple, the restriction of the Killing form of $\mathrm{SO}(5)$ to $\mathrm{SO}(4)$ turns out to be a multiple of the Killing form (i.e., the same multiple on each 
irreducible factor). Then the general results of this section applies also for this case. We have proved that the (autoparallel) $\mathrm{SO}(n+1)$-invariant distribution $\tilde{\mathfrak{p}}^{\prime}$ is contained in the symmetric distribution $\mathfrak{s}$ of $M$.

We will prove the equality, or equivalently, from Lemma 6.2, that

$$
(\mathrm{SO}(n+2) / \mathrm{SO}(n),\langle\cdot, \cdot\rangle)
$$

is not a locally symmetric space.

It is not difficult to see that, up to rescaling, $(\mathrm{SO}(n+2) / \mathrm{SO}(n),\langle\cdot, \cdot\rangle)$ is the unit tangent bundle of the sphere of curvature 2 (with the Sasaki metric). From the following remark we obtain that this space is not locally symmetric.

Remark 6.4. The unit tangent bundle $M^{2 n-1}$ to the sphere of curvature 2 is never locally symmetric. In fact, assume $n=2$. Then $\mathrm{SO}(3)$ acts simply transitively on $M^{3}$. The universal cover $\tilde{M}^{3}$ of $M^{3}$ is compact and diffeomorphic to $\operatorname{Spin}(3)$. If $\tilde{M}^{3}$ is symmetric then it must be irreducible. Moreover, $\tilde{M}^{3}$ must be of rank one. In fact the isotropy representation is an irreducible and polar representation acting in a 3-dimensional space (which implies that is transitive on the unit sphere). Then all geodesics in $\tilde{M}^{3}$ have are closed of the same length. Then all geodesics of $M^{3}$ would admit a common period. But any horizontal geodesic of $M^{3}$ has length $\sqrt{2} \pi$. But a vertical geodesic has length $2 \pi$, which is not rationally related to the length of any horizontal geodesic. Then $M^{3}$ is not locally symmetric. Now observe that $M^{3}$ is in a canonical way a totally geodesic submanifold of $M^{2 n-1}$. Then $M^{2 n-1}$ is not locally symmetric. Otherwise, $M^{3}$ would be locally symmetric.

Observe that the unit tangent bundle to the sphere of dimension 2 and curvature 1 is a Lie group with a bi-invariant metric and hence a symmetric space.

Remark 6.5. Notice the difference between the above example and taking $k=$ 2 in Example 4.10. In both cases the underlying differentiable manifold is the Stiefel manifold $\mathrm{SO}(n+2) / \mathrm{SO}(n)$, but in Example 6.3 the metric is not normal homogeneous. Recall that in Example 4.10, with the normal homogeneous metric, the leaf of symmetry is the circle $S^{1}$ (and hence, it is of the group type).

Example 6.6. Let us consider the standard inclusions $\mathrm{SU}(3) \supset \mathrm{SO}(3) \supset \mathrm{SO}(2)$. Then $M=\mathrm{SU}(3) / \mathrm{SO}(2)$, with the metric above defined has index of symmetry equal to 2 and leaf of symmetry the sphere $S^{2}$. In fact, let us check that $M$ is not a symmetric space. Recall that $M$ is the Aloff-Wallach manifold $M=W_{1,-1}^{7}$, and hence $H^{4}(M, \mathbb{Z})=0$, according with AW75, Lemma 3.3]. Assume that $M$ is a symmetric space, then must be one of the following: $S^{7}, S^{5} \times S^{2}, S^{4} \times S^{3}$, or $S^{3} \times S^{2} \times S^{2}$. The last two cases are excluded by the restriction on the cohomology. It cannot be $M=S^{7}$, since $\mathrm{SU}(3)$ is not transitive on $S^{7}$. Finally, if $M=S^{5} \times S^{2}$, then, projecting down to the second factor, we would obtain an isometric action of $\mathrm{SU}(3)$ on $S^{2}$. Such an action must be trivial since $\mathrm{SU}(3)$ is simple and $\operatorname{dim} \mathrm{SU}(3)>$ $\operatorname{dim} \mathrm{SO}(3)$. So, $\mathrm{SU}(3)$ cannot be transitive on $M$, which is a contradiction. With a similar argument, we can prove that $M$ is an irreducible Riemannian manifold.

Example 6.7 (The Wallach 24-manifold). Consider $F_{4} \supset \operatorname{Spin}(9) \supset \operatorname{Spin}(8)$. Notice that $F_{4} / \operatorname{Spin}(9)$ is the Cayley plane. The manifold $W^{24}=F_{4} / \operatorname{Spin}(8)$ is the so-called Wallach manifold and it has leaf of symmetry $S^{8}=\operatorname{Spin}(9) / \operatorname{Spin}(8)$. In fact, it is well-known that $W^{24}$ is topologically different from a symmetric space. Recall that in this case $W^{24}$ can be endowed with a metric of positive curvature. 


\section{REFERENCES}

[AleEM] D. V. Alekseevskii (originator), Reductive space, Encyclopedia of Mathematics, http: //www.encyclopediaofmath.org/index.php?title=Reductive_space\&oldid=11232

[AW75] S. Aloff and N. Wallach, An infinite family of distinct 7-manifolds admitting positively curved Riemannian structures, Bull. Amer. Math. Soc. 81 (1975), 93-95.

[Car26] É. Cartan, Sur une classe remarquable d'spaces de Riemann. I, II, Bull. Soc. Math. France 54 (1926), 214-264, 55 (1927), 114-134.

[BCO03] J. Berndt, S. Console, and C. Olmos, Submanifolds and holonomy, Research Notes in Mathematics, vol. 434, Chapman \& Hall/CRC, Boca Raton, 2003.

[EO94] J.-H. Eschenburg and C. Olmos, Rank and symmetry of Riemannian manifolds, Comment. Math. Helv. 69 (1994), no. 3, 483-499.

[Kos56] B. Kostant, On differential geometry and homogeneous spaces. II, Proc. Natl. Acad. Sci. USA 42 (1956), 354-357.

[OR12a] C. Olmos and S. Reggiani, The skew-torsion holonomy theorem and naturally reductive spaces, J. Reine Angew. Math. 664 (2012), 29-53.

[OR12b] , A note on the uniqueness of the canonical connection of a naturally reductive space, arXiv:1210.8374v1 [math.DG] (2012).

[Reg10] S. Reggiani, On the affine group of a normal homogeneous manifold, Ann. Global Anal. Geom. 37 (2010), no. 4, 351-359.

[Reg13] _ A Berger-type theorem for metric connections with skew-symmetric torsion, J. Geom. Phys. 65 (2013), 26-34.

Facultad de Matemática, Astronomía y Física, Universidad Nacional de Córdoba, Ciudad Universitaria, 5000 Córdoba, Argentina

E-mail address: olmos@famaf.unc.edu.ar, reggiani@famaf.unc.edu.ar

Department of Mathematics, Hiroshima University, 1-3-1 Kagamiyama, Higashi-HiroSHIMA, 739-8526, JAPAN

E-mail address: tamaru@math.sci.hiroshima-u.ac.jp 